\title{
A Magnetic Field Separation Technique for a Scaled Model Ship through an Earth's Magnetic Field Simulator
}

\author{
Hyun-Ju Chung*, Chang-Seob Yang, and Woo-Jin Jung \\ 6th R\&D Institute-3, Agency for Defense Development, Changwon 645-600, Korea
}

(Received 30 September 2014, Received in final form 27 October 2014, Accepted 27 October 2014)

\begin{abstract}
This paper presents an experimental technique to accurately separate a permanent magnetic field and an induced one from the total magnetic fields generated by a steel ship, through compensating for the Earth's magnetic field. To achieve this, an Earth's magnetic field simulator was constructed at a non-magnetic laboratory, and the field separation technique was developed, which consisted of five stages. The proposed method was tested with a scaled model ship, and its permanent and induced magnetic fields were successfully extracted from the magnetic field created by the ship. Finally, based on the separated permanent magnetic field data, the permanent magnetization distribution on the hull was predicted by solving an inverse problem. Accordingly, the permanent magnetic fields generated by the ship can easily be calculated at any depth of water.
\end{abstract}

Keywords : Earth's magnetic field simulator, induced magnetization, inverse problem, permanent magnetization

\section{Introduction}

A naval ship is constructed of ferrous steel plates. When the ship is placed in the Earth's magnetic field, a local anomaly of underwater magnetic fields, due to the induced and the permanent magnetizations distributed on the hull, is created around the ship. Even though its magnitude is much smaller than that of the Earth's magnetic field, it can activate underwater weapon systems equipped with high-sensitivity magnetic sensors, such as influence mines, or underwater detecting equipment. To protect the ship from these threats, it is necessary to establish proper countermeasures against the detection sensors of an influence mine or an underwater detection system [1-9].

A ship made of ferromagnetic material generates two underwater field components, which consist of an induced magnetic field due to the induced magnetization, and a permanent magnetic field due to the permanent magnetization. In general, for the purpose of reducing the underwater magnetic field of a naval ship, a deperming process is first conducted to eliminate permanent magnetization distributed on the hull; and then, a degaussing process is

(C)The Korean Magnetics Society. All rights reserved.

*Corresponding author: Tel: +82-10-4542-8441

Fax: +82-55-542-3737, e-mail: hyunju30@add.re.kr performed to minimize the field due to induced magnetization, as well as residual permanent magnetization after the deperming process [7].

However, it is very difficult to predict the field disturbance due to the permanent magnetization, because of the absence of information about the magnetic history of the hull, such as the mechanical or thermal stress. The only possible way is to predict the permanent magnetization distribution on the hull through solving an inverse problem with measured underwater field data, and to establish a proper deperming process based on the calculated permanent magnetization. Therefore, an accurate measurement method on the induced and permanent magnetic fields is required, in order to more effectively perform the magnetic treatment, which consists of deperming and degaussing processes. This is because a successful magnetic silencing of the ship strongly depends on how to minimize the individual field components produced by different magnetic sources of the induced magnetization and permanent magnetization of the hull. In our previous works [8, 9], the induced magnetic field was first obtained by means of a commercial electromagnetic (EM) software package, based on the finite element method (FEM). Then, the permanent magnetic field was analogized, by subtracting the induced magnetic field from measurement data of underwater magnetic fields under the Earth's magnetic field. However, this approach has a limit on the accuracy 
of numerical prediction of the induced magnetic field, because it is very hard to build a three-dimensional finite element model of a real ship, which contains complicated internal structures, such as bulkheads, engines, and various steel-made equipment.

In this paper, an experimental technique is proposed to accurately separate the induced and permanent magnetic fields from the measured underwater field data. For the purpose of doing this, an Earth's magnetic field simulator was built to realize the environment of the Earth's magnetic field at any location of the world; and accordingly, the field separation technique was developed, which consisted of five main stages. To verify the proposed method, a scaled model ship was tested, and its permanent and induced magnetic field components were successfully extracted from the total magnetic field that was measured. Moreover, a permanent magnetization distribution on the hull was predicted, by solving an inverse problem, based on the material sensitivity analysis presented in our previous work [10]. Finally, the permanent magnetic field generated by the ship at any depth of water can be easily calculated from the permanent magnetization distribution obtained by the inverse problem.

\section{Basic Properties of Underwater Magnetic Fields}

In this section, basic properties of underwater magnetic fields generated from the ship are briefly explained for a better understanding of the goal of this paper. As described earlier, the induced and permanent magnetization distributed on the ferromagnetic hull causes a local perturbation of underwater magnetic fields. According to the field-generating magnetization type, the perturbation field is decomposed into the induced and permanent magnetic fields, which belong to the magneto-static field.

Consider this perturbation field from the viewpoint of Maxwell's equations. When a ferromagnetic hull is placed in the Earth's magnetic field $\mathbf{H}_{o}$, a local perturbation field $\mathbf{H}_{\text {red }}$, expressed in terms of the magnetic scalar potential $\phi_{m}$, is created around a ship.

$$
\mathbf{H}=\mathbf{H}_{o}+\mathbf{H}_{\text {red }}=\mathbf{H}_{o}-\nabla \phi_{m}
$$

The field $\mathbf{H}_{\text {red }}$ is due to the hull magnetization $\mathbf{M}$, which is the sum of the induced magnetization $\mathbf{M}_{\text {ind }}$, and the permanent magnetization $\mathbf{M}_{\text {rem }}$.

$$
\mathbf{M}=\mathbf{M}_{\text {ind }}+\mathbf{M}_{\text {rem }}
$$

The constitutive relation between $\mathbf{B}$ and $\mathbf{H}$ is written as:

$$
\mathbf{B}=\mu_{o}(\mathbf{H}+\mathbf{M})
$$

If the hull thickness is relatively small and its permeability is high, the direction of the magnetization can be considered parallel to the hull surface, and its magnitude can also be assumed constant $[3,4]$.

When the magnetization distribution on the hull is given under the above assumption, the perturbation field is easily calculated by:

$$
\mathbf{H}_{r e d}=\frac{1}{4 \pi} \int_{V} \frac{3(\mathbf{M} \cdot \mathbf{r}) \mathbf{r}-r^{2} \mathbf{M}}{r^{5}} d V=-\frac{1}{4 \pi} \int_{S} \mathbf{M} \cdot \mathbf{n} \frac{r}{r^{3}} d S
$$

where, $V$ is the volume enclosed by the hull surface $S, r$ is the distance between an observation point and the hull magnetization, and $\mathbf{n}$ is the unit vector normal to the hull surface [4].

\section{Separation Technique for an Underwater Magnetic Field}

In this section, an Earth's magnetic field simulator is explained in detail. It was designed and built for the purpose of compensating for the Earth's magnetic field. Then, an accurate extraction method of permanent and induced magnetic fields from measured underwater field data is described.

\subsection{Earth's magnetic field simulator}

The Earth's magnetic field simulator can compensate

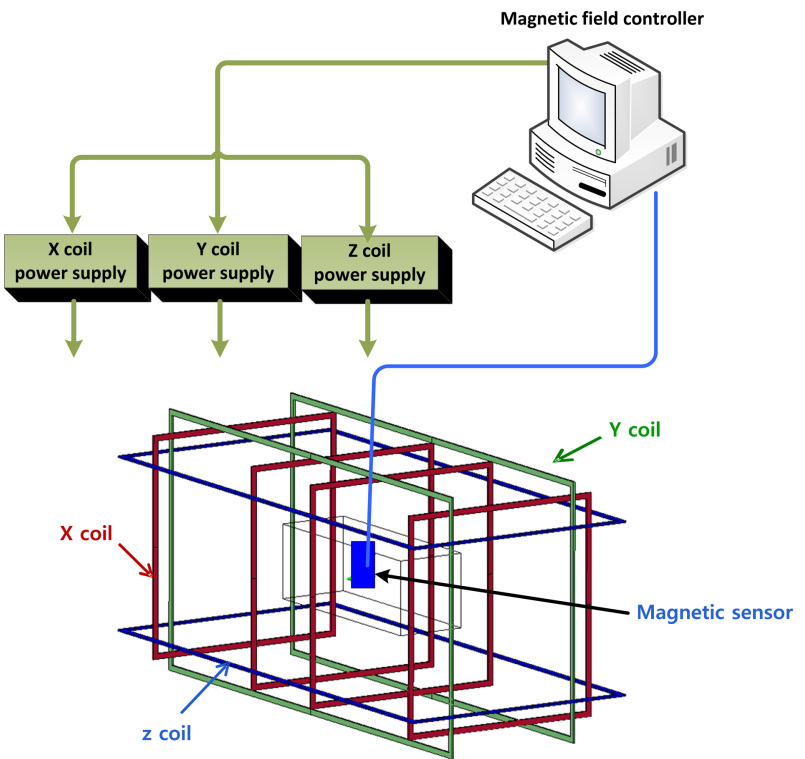

Fig. 1. (Color online) Schematic diagram of an Earth's magnetic simulator, consisting of a tri-axial magnetic field generation coil, power supplies, a magnetic sensor, and a magnetic field controller. 
for the Earth's magnetic field at a measurement station, and realize an environment of the Earth's magnetic field in a certain region any wherein the world where a ship is located. Figure 1 shows a schematic diagram of the Earth's magnetic field simulator developed by the Agency for Defense Development. It is composed of four major parts: The first is tri-axial magnetic field generation coils to produce a magnetic field; the second is power supplies to provide electric currents to the field generation coils; the third is a magnetic sensor to measure the magnetic field at the center of the field simulator; and the last is a magnetic field controller to adjust the electric currents applied to individual field generation coils.

The tri-axial magnetic field generation coils consist of eight coils (four X coils, of which the area is normal to the south-north direction; two Y coils normal to the eastwest direction; and two $\mathrm{Z}$ coils normal to the vertical direction). Table 1 presents detailed specifications of the coils. The X coils have two inner coils with 27 turns, and two outer coils with 95 turns, to enhance the field uniformity in the longitudinal (south-north) direction. The $\mathrm{X}$ coils are connected in series. The Y coils have two coils with 88 turns, and the $Z$ coils have two coils with 66 turns. These $\mathrm{Y}$ and $\mathrm{Z}$ coils are also connected in series.

To precisely control a magnetic field value at the center of the field simulator, we first measured the individual coil effects, which correspond to the magnetic field values produced by each coil when an electric current of $1 \mathrm{~A}$ is applied. The $\mathrm{X}$ coil effect was $11.15 \mu \mathrm{T} / \mathrm{A}$, the $\mathrm{Y}$ coil effect was $13.20 \mu \mathrm{T} / \mathrm{A}$, and the $\mathrm{Z}$ coil effect was 11.75 $\mu \mathrm{T} / \mathrm{A}$. The Earth's magnetic fields at the measurement station (Jinhae region), where the Earth's magnetic field simulator was installed, are $31.5 \mu \mathrm{T}$ in the south-north direction $(\mathrm{Bx})$, and $38.5 \mu \mathrm{T}$ in the vertical direction(Bz). In order to compensate for the Earth's magnetic fields at the measurement station, currents of $-2.83 \mathrm{~A}$ and $-3.28 \mathrm{~A}$ must be fed to the $\mathrm{X}$ and $\mathrm{Z}$ coils, respectively.

Figure 2 shows the variation of magnetic fields measured at the center of the measurement station, as each coil current increases. As seen in the figure, the field magnitude is exactly proportional to the coil current. The

Table 1. Specific specification of a tri-axial magnetic field generation coil.

\begin{tabular}{cccc}
\hline \hline & North to South & East to West & Vertical \\
\hline Coil size & $(6 \times 6) \mathrm{m}$ & $(14.4 \times 7) \mathrm{m}$ & $(15 \times 7) \mathrm{m}$ \\
Coil turns & Inner coil $(27)$ & 88 & 66 \\
& Outer coil(95) & & \\
Coil resistance $(\Omega)$ & 12.1 & 11.8 & 11.5 \\
Coil effect $(\mathrm{nT} / \mathrm{A})$ & 11,146 & 13,199 & 11,753 \\
\hline
\end{tabular}

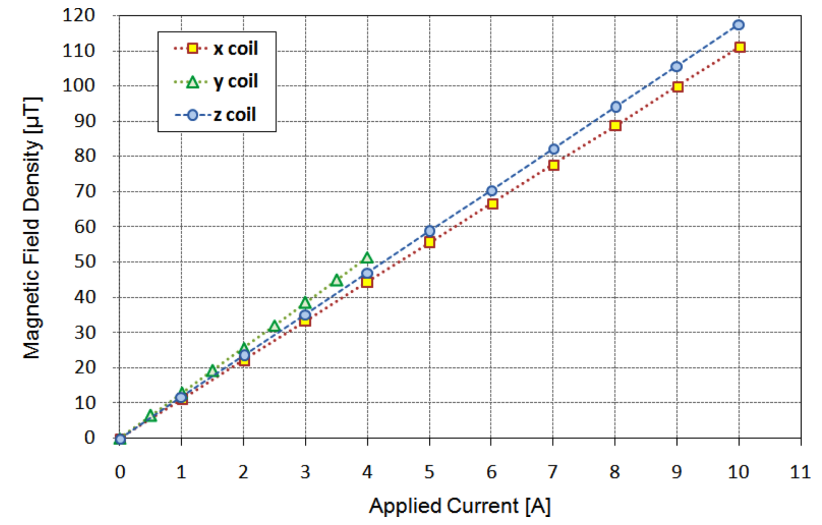

Fig. 2. (Color online) Magnetic field measured in the middle of each coil, according to the current applied to the tri-axial magnetic field generation coil.

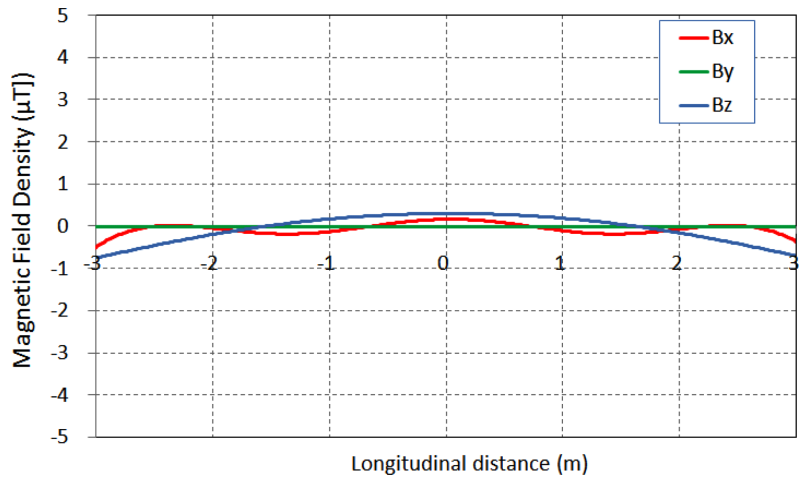

Fig. 3. (Color online) Residual fields at the longitudinal line of the south-north direction, after compensation for the Earth's magnetic field at the measurement station.

$\mathrm{X}$ and $\mathrm{Z}$ coils generate magnetic fields up to more than $110 \mu \mathrm{T}$. Thus, the field simulator capacity is enough to remove the Earth's magnetic field at the measurement station.

Figure 3 shows three components of the residual fields along the longitudinal (south-north) direction, after the field compensation. Figures 4, 5 and 6 show the distribu-

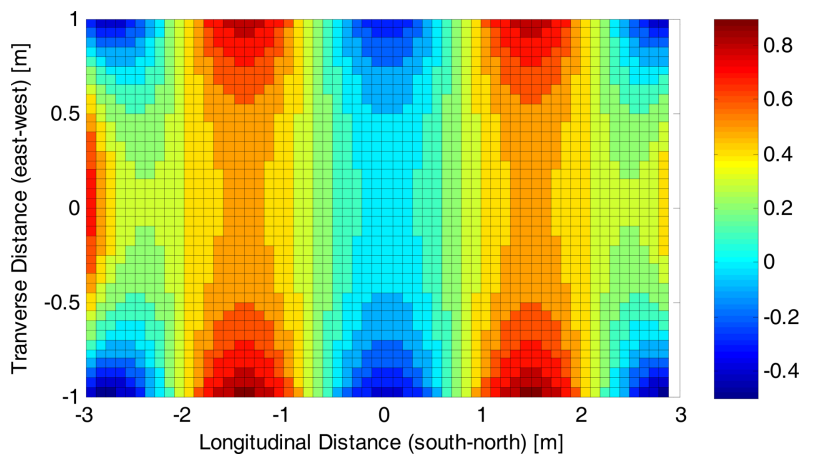

Fig. 4. (Color online) Distribution of residual fields in a northern direction $(\mathrm{Bx})$. 


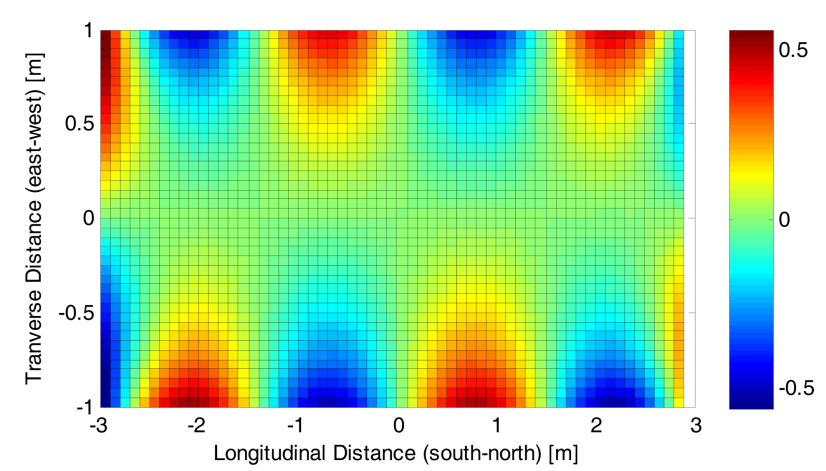

Fig. 5. (Color online) Distribution of residual fields in an eastern direction (By).

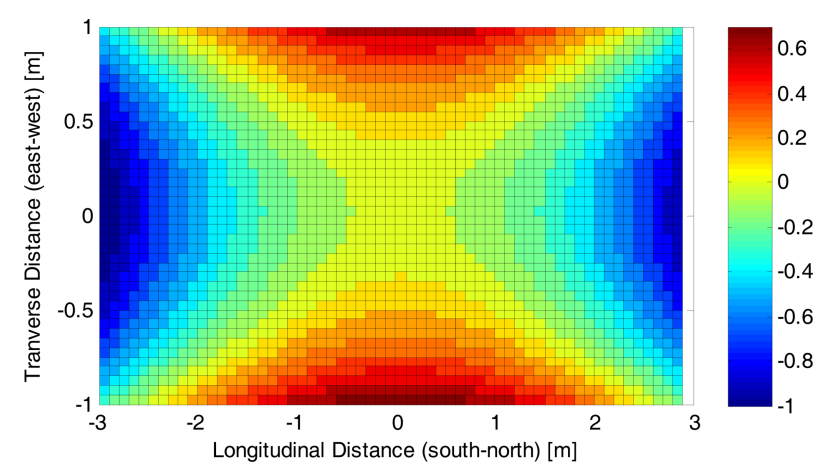

Fig. 6. (Color online) Distribution of residual fields in a vertical direction toward the ground $(\mathrm{Bz})$.

tion of residual fields in a cross-sectional area of $6 \mathrm{~m}$ (south-north) $\times 2 \mathrm{~m}$ (east-west). These figures show that the maximum residual field value for the northern direction $(\mathrm{Bx})$ is $-0.43 \mu \mathrm{T}$, the maximum residual field value for the western direction (By) is $-0.59 \mu \mathrm{T}$, and the maximum residual field value for the vertical direction (Bz) is $-0.72 \mu \mathrm{T}$. The maximum residual field in the area is reduced by more than $98 \%$ of the Earth's magnetic field, before compensation.

\subsection{Separation of induced or permanent fields}

The fields measured from a scaled ship after compensation for the Earth's magnetic field correspond to the field due only to the permanent magnetization distributed on the hull. Therefore, through using the field simulator, the permanent magnetic field generated from the ship can be accurately measured. Then, the induced magnetic field is easily extracted, by subtracting the permanent magnetic field from the measured field, before compensation for the Earth's magnetic field.

The separation processes of the permanent or induced magnetic fields from the magnetic field generated from the ship comply with the following steps that Fig. 7 presents. First, the individual coil effects are measured

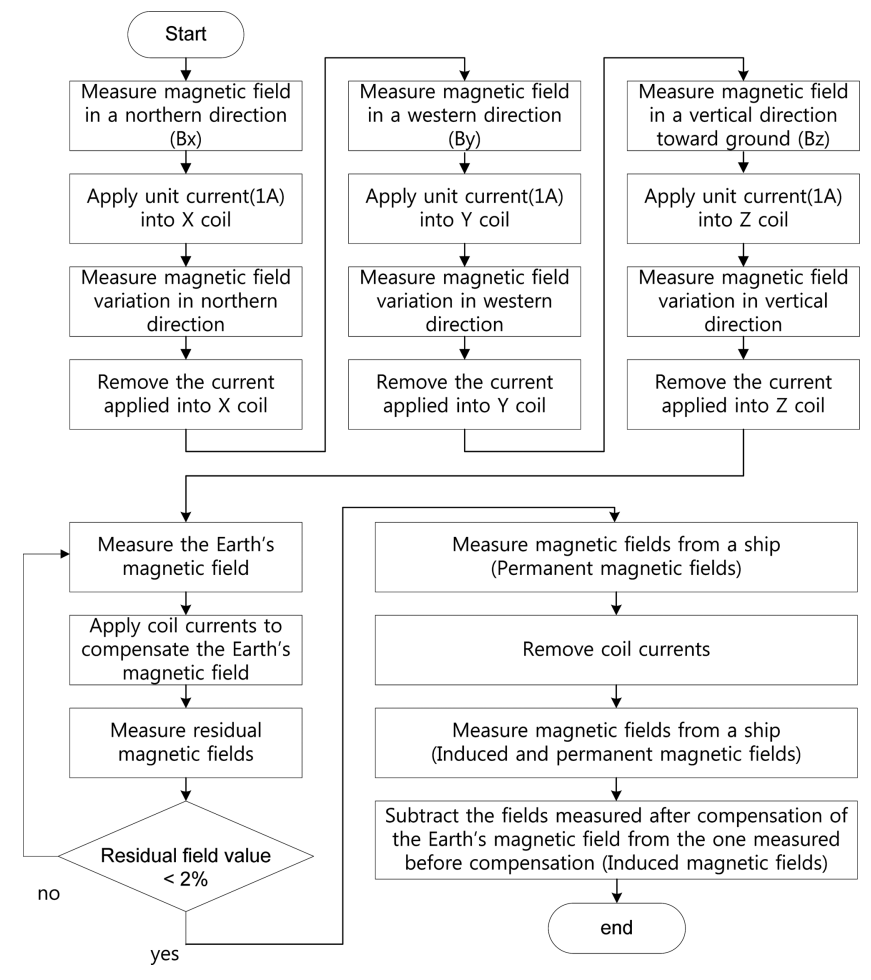

Fig. 7. A flow chart for separating permanent and induced magnetic fields, through compensation for the Earth's magnetic field.

one-by-one, as seen in the upper part of the figure. Second, the Earth's magnetic field at the measurement station is measured, and coil currents adequate to compensate for the Earth's magnetic field are applied. The later process is repeated, until the residual field after compensation is less than $2 \%$ of the Earth's magnetic field before compensation. Third, the magnetic field from the ship after field compensation is measured. Its value exactly corresponds to the permanent magnetic field. Fourth, the currents applied to the field generation coils are removed, and then the field from the ship under the Earth's magnetic field is measured. This is the sum of the permanent and induced magnetic fields. Finally, we subtract the field measured after compensation, from the one measured before compensation. The resultant corresponds to the induced magnetic field.

\section{Results}

\subsection{Experimental setup}

Figure 8 shows a scaled model ship used for separating the permanent and induced magnetic fields. It is made out of ferrous steel plates with upper deck thickness of 0.3 $\mathrm{mm}$, and lower deck thickness of $0.6 \mathrm{~mm}$. The relative permeability of the steel plates is considered to be 160 . 


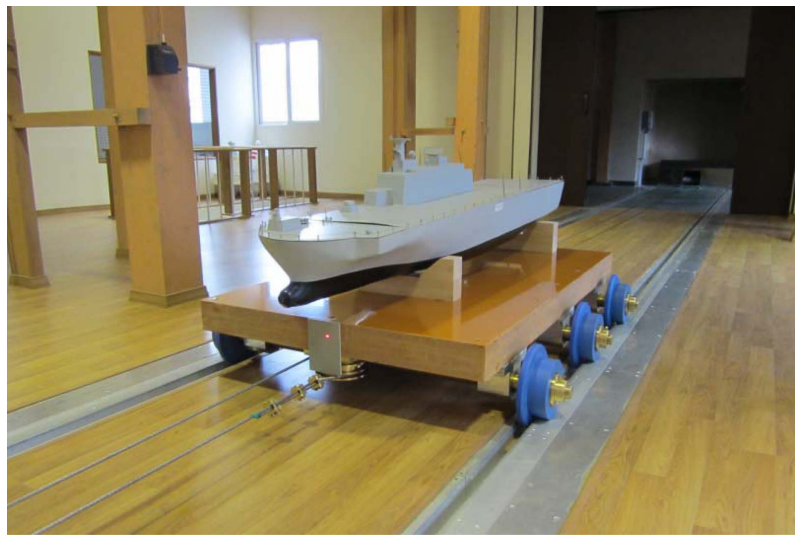

Fig. 8. (Color online) Photograph of the scale model ship.

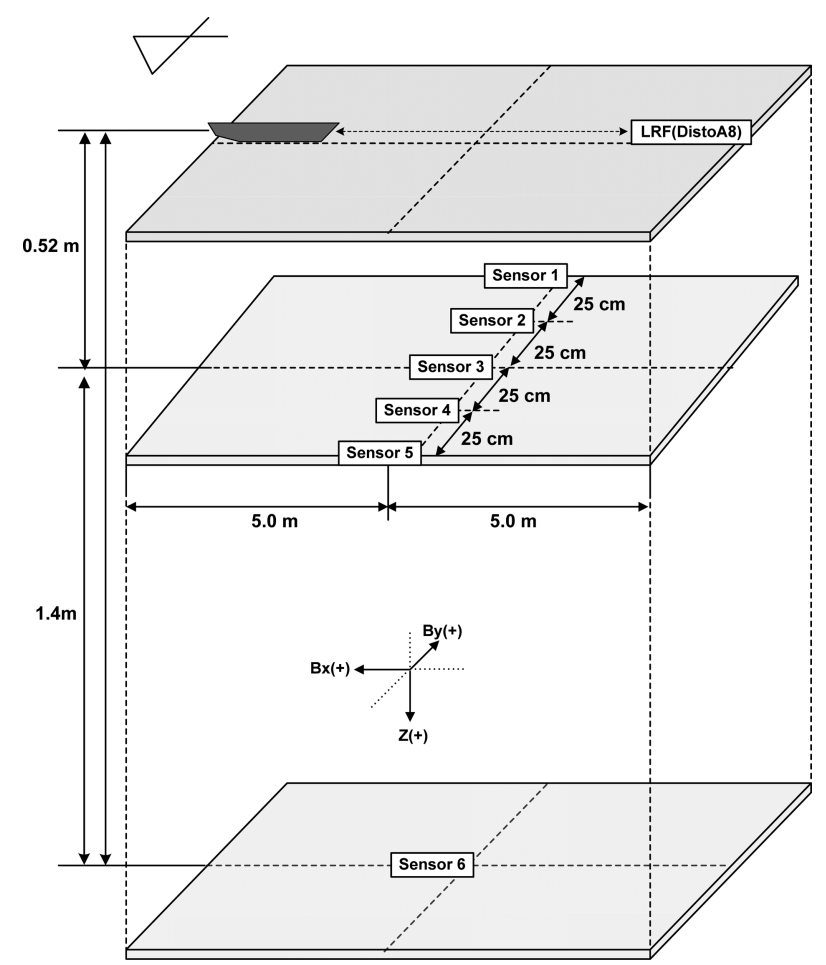

Fig. 9. Experimental setup.

The ship is $2.4 \mathrm{~m}$ long, $0.5 \mathrm{~m}$ wide, and $0.25 \mathrm{~m}$ high. The magnetic fields were measured with six tri-axial magnetic sensors (Bartington Instruments, Mag03MS70) located under the ship, as Fig. 9 shows. At the experimental station, $\mathrm{Bx}$ is set to the northern direction, By to the eastern direction, and $\mathrm{Bz}$ to the vertical direction. In this experiment, instead of using many sensors, the ship slowly moves toward the south or the north along the longitudinal centerline, against the sensors at a standstill. As mentioned earlier, the Earth's magnetic field at the experimental station (Jinhae) is $31.5 \mu \mathrm{T}$ in the south-north direction (Bx) and $38.5 \mu \mathrm{T}$ in the vertical direction (Bz).

The model ship's locations from the magnetic sensors

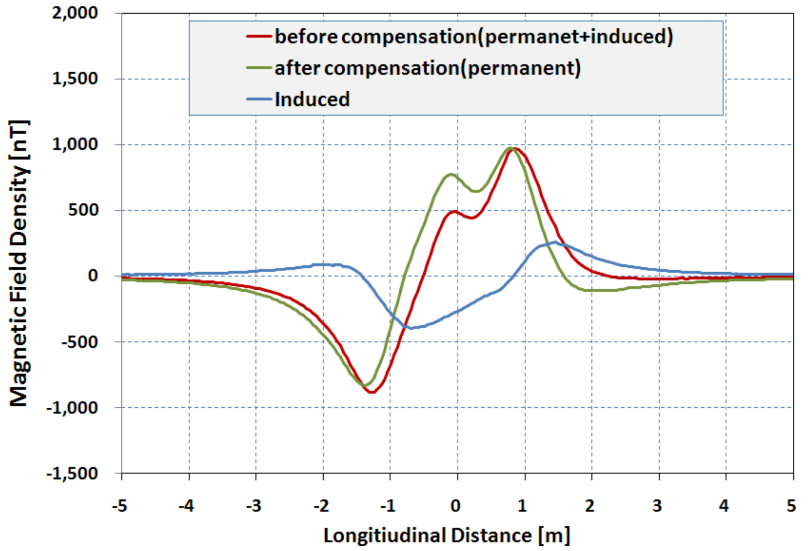

Fig. 10. (Color online) Horizontal magnetic fields $(\mathrm{Bx})$ measured at a line parallel to the x-axis and a depth of $0.52 \mathrm{~m}$ below the keel line, when the model ship is headed toward the north.

were measured very accurately with Laser Range Finder, DistoA8 (LRF), which has a distance measurement error of less than $1 \mathrm{~mm}$, to avoid the phase difference of the measuring data at each measurement stage.

\subsection{Induced or permanent fields measured from thes- caled model ship}

Figures 10 and 11 show the horizontal (x-axis) and vertical (z-axis) magnetic fields measured on the longitudinal centerline parallel to the $\mathrm{x}$-axis, and at a depth of $0.52 \mathrm{~m}$ below the keel line (sensor 3 in Fig. 9), when the ship is headed toward the north. Figures 12 and 13 present the magnetic fields measured on the centerline at a depth of $0.52 \mathrm{~m}$ below the keel line (sensor 3 in Fig. 9), when the ship is headed toward the south. From these figures, we can compare the permanent magnetic fields

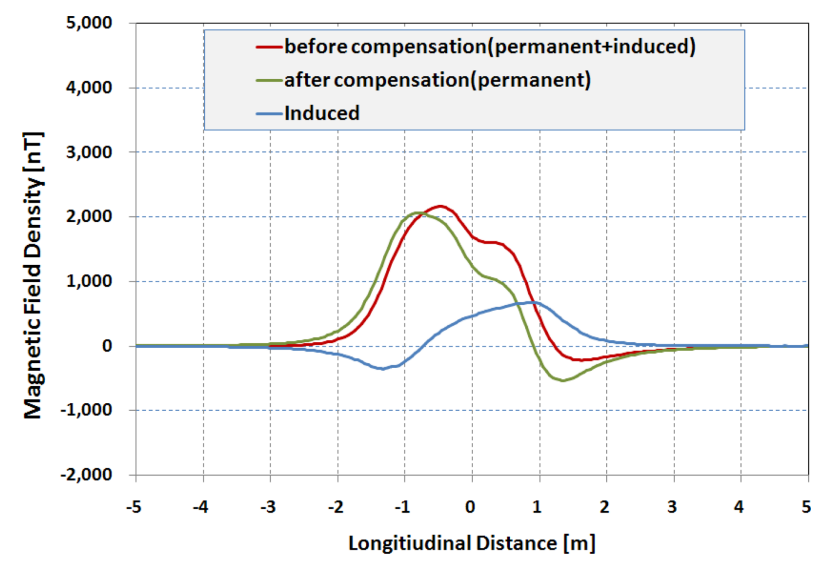

Fig. 11. (Color online) Vertical fields (Bz) measured at a line parallel to the $\mathrm{x}$-axis and a depth of $0.52 \mathrm{~m}$ below the keel line, when the model ship is headed toward the north. 


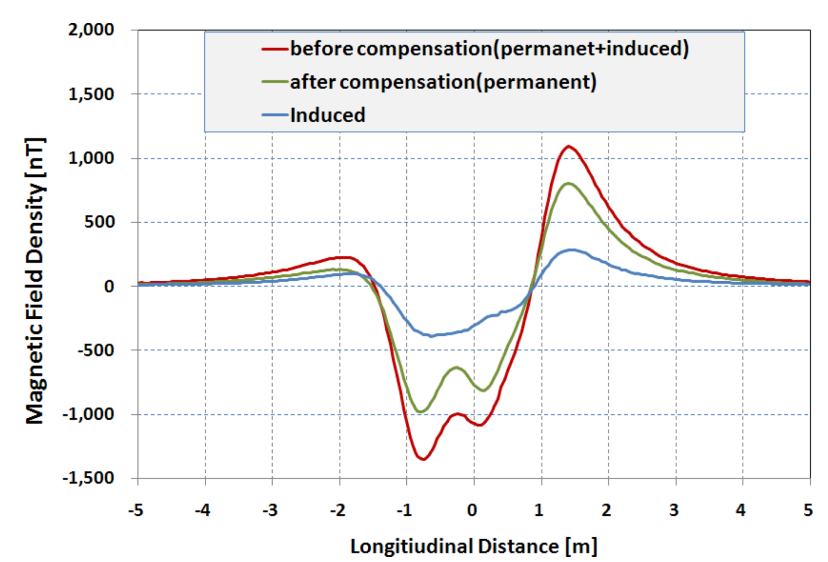

Fig. 12. (Color online) Horizontal magnetic fields $(\mathrm{Bx})$ measured on a line parallel to the axis at the depth of $0.52 \mathrm{~m}$ below the keel line, when the model ship is headed toward the south.

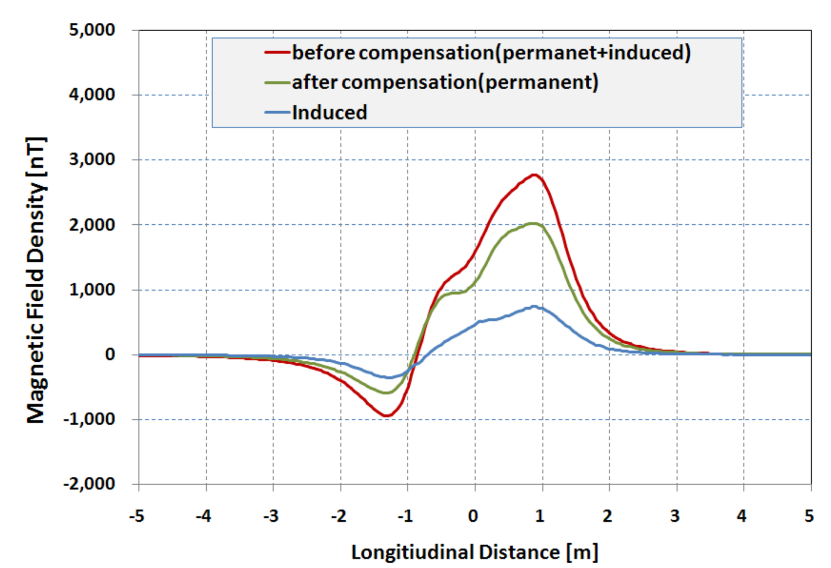

Fig. 13. (Color online) Vertical magnetic fields (Bz) measured at a line parallel to the axis at a depth of $0.52 \mathrm{~m}$ below the keel line, when the model ship is headed toward the south.

with the induced magnetic fields, in terms of shape and magnitude.

In Figs. 10 and 11, it is observed that the permanent and induced fields are different in shape and magnitude from each other when the ship heads north. On the other hand, in Figs. 12 and 13, where the ship heads south, the shapes of the permanent fields are similar to those of the induced fields, even though their magnitudes differ from each other. From this result, it is inferred that the direction of permanent magnetization distributed on the hull is aligned toward the stern. That is because the direction of induced magnetization on the hull must be the same as that of the Earth's magnetic field that appears at the measurement station (i.e. in the south-north direction), when the ship steers southward. Moreover, comparison between Figs. 10 to 13 reveals that the induced magnetic fields have similar shapes and magnitudes, because the Earth's magnetic field applied is headed toward the south-north direction, regardless of the ship's heading direction. However, permanent magnetic fields have the same shapes and magnitudes of which the directions are reversed, according to the ship's heading direction. That is because the direction of permanent magnetization on the hull does not change depending on the direction of ship movement, or the Earth's magnetic field that is applied.

To protect a ship against underwater safety hazards, it is necessary to accurately predict the magnetic fields generated from a ship at any depth in seawater. Based on the proposed field separation technique, it is now possible to obtain accurate field computation results of the induced and permanent magnetic fields. The induced magnetic field is easily calculated with commercial FEM software packages only when numerical modeling parameters, specifically on the relative permeability value, are properly tuned. On the other hand, it is still very cumbersome to compute the permanent magnetic field underwater, because there is no information about the history of permanent magnetization distributed on the hull. In order to efficiently deal with this problem, the permanent magnetization $\mathbf{M}_{r e m}$ distributed on the hull is first obtained by solving an inverse problem analysis method, based on the material sensitivity analysis in Ref. [10], and permanent magnetic field data measured at a certain depth of water. Then, the permanent magnetic field at the desired depth of water is calculated with the obtained permanent magnetization distribution. To achieve this, the hull surface shown in

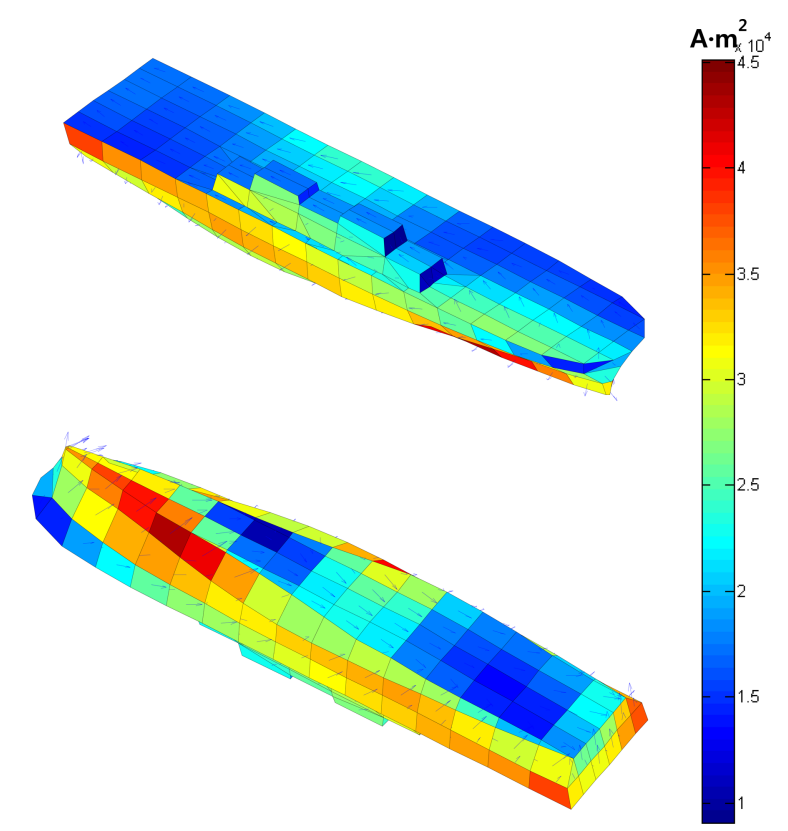

Fig. 14. (Color online) Equivalent permanent magnetization moment distributed on the hull of the scaled model ship, after solving the inverse problem. 


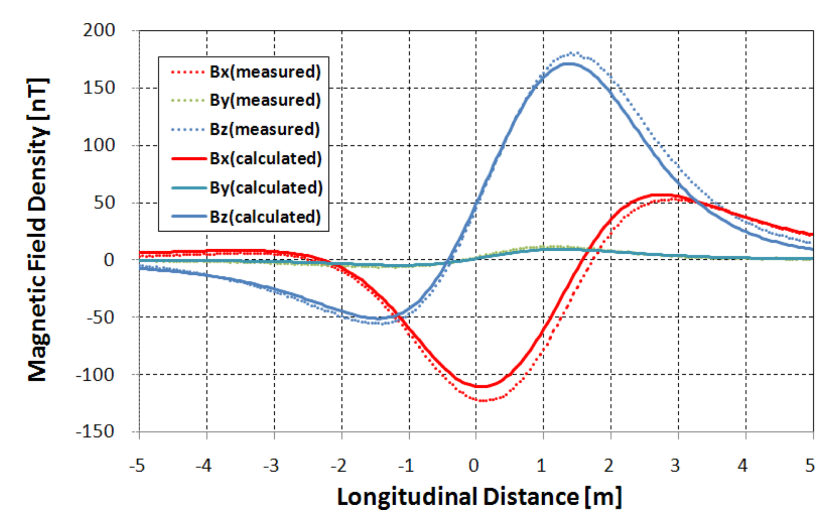

Fig. 15. (Color online) Comparison results between the calculated magnetic fields and the measured one, at a line parallel to the $\mathrm{x}$-axis, and a depth of $1.92 \mathrm{~m}$ below the keel line of the model ship.

Fig. 14 was divided into 322 sheet elements, where an optimum distribution of equivalent magnetic dipole moments was sought out. The field data measured with the installed magnetic sensors (sensor 1 to sensor 5 in Fig. 9) at a depth of $0.52 \mathrm{~m}$ under the ship was considered as target fields for an inverse problem. An objective function of the problem is mathematically defined as follows.

$$
F=\sum_{i=1}^{n} \sum_{j=1}^{3}\left(B_{i j}^{k}-B_{o i j}\right)^{2}
$$

where $n$ is the total measurement points of $505, B_{i j}^{k}$ is the $j$ th component of the magnetic flux density at the $i$ th measurement point, which is predicted based on an intermediate permanent magnetization distribution obtained in the $k$ th iterative calculation process, and $B_{o i j}$ is the target fields measured at the measurement points.

After solving the inverse problem, Fig. 14 illustrates an optimum distribution of the equivalent magnetization moment vectors over the hull, where the direction and magnitude of the magnetization vector is expressed in terms of arrows and contour colors, respectively. Figure 15 compares the permanent magnetic field calculated from the optimum magnetization distribution, and the measured one, at a depth of $1.92 \mathrm{~m}$ (sensor 6 in Fig. 9) below the keel line of the model ship. As a result, the predicted permanent magnetic field components show good agreement with the measured ones, within less than
$10 \%$ of the difference in maximum field values.

\section{Conclusion}

In this paper, an experimental technique was proposed to accurately extract the permanent and induced magnetic fields from the total field generated by a steel ship. To achieve the goal, an Earth's magnetic field simulator was built, and accordingly the field separation procedure was developed, which consisted of five main stages. To verify the proposed method, a scaled model ship was tested, and the permanent and induced magnetic fields were successfully extracted from the total magnetic field created by the ship. Moreover, based on the separated permanent magnetic field data, the permanent magnetization distribution on the hull was predicted. Accordingly, the permanent magnetic field generated by the ship can then be calculated at any depth of water. Finally, we infer that the proposed method will be very useful in establishing proper countermeasures for reducing underwater magnetic fields.

\section{References}

[1] John J. Holmes, Exploitation of A Ship's Magnetic Field Signatures, Morgan \& Claypool, (2006), pp. 1-59.

[2] R. Donati and J. P. Le Cadre, IEE Proc. Radar Sonar Navig. 149, 221 (2002).

[3] O. Chadebec, J. Coulomb, J. Bongiraud, G. Cauffet, and P. Thiec, IEEE Trans. Magn. 38, 1005 (2002).

[4] O. Chadebec, J. Coulomb, G. Cauffet, and J. Bongiraud, IEEE Trans. Magn. 39, 1634 (2003).

[5] N. Choi, G. Jeung, C. Yang, H. Chung, and D. Kim, IEEE Trans. Appl. Supercond. 42, 4904504 (2012).

[6] N. Choi, G. Jeung, S. Jung, C. Yang, H. Chung, and D. Kim, IEEE Trans. Magn. 48, 419 (2012).

[7] H. Liu and Z. Ma, Proc. Int. Conf. Mechatronics and Automation 3133 (2007).

[8] C. Yang, K. Lee, G. Jung. H. Chung, J. Park, and D. Kim, J. Appl. Physics 103, 905 (2008).

[9] K. Lee, G. Jeung, C. Yang, H. Chung, J. Park, H. Kim, and D. Kim, IEEE Trans. Magn. 45, 1478 (2009).

[10] N. Choi, D. Kim, C. Yang, H. Chung, H. Kim, and D. Kim, Journal of Electrical Engineering \& Technology 6, 799-805 (2011). 